\title{
Malignant GIST masquerading as a bleeding duodenal diverticulum
}
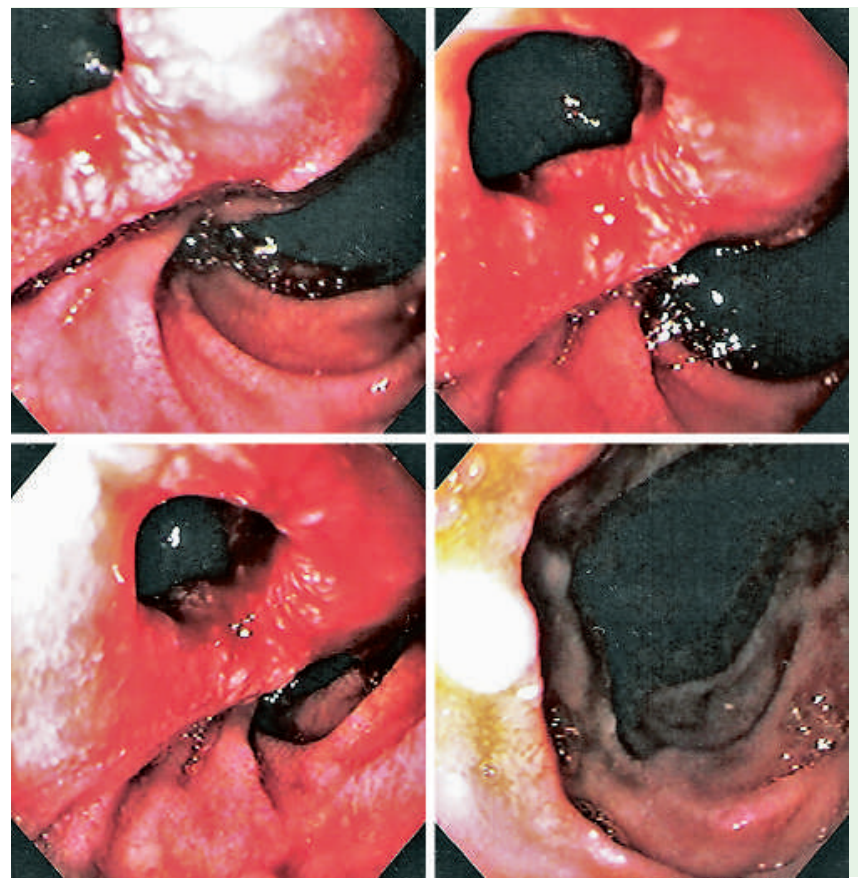

Figure 1 Endoscopic images revealed what appeared to be a diverticulum at the junction of the second and third part of the duodenum, with evidence of large amount of clot within.

A 63-year-old man was accepted in transfer with a diagnosis of gastrointestinal bleeding from a duodenal diverticulum. An apparent bleeding diverticulum was visualized on repeat endoscopy at the junction of the second and third part of the duodenum ( $\bullet$ Figure 1 ). Computed tomography scan revealed a large (15 $\times 10 \mathrm{~cm}$ ) heterogeneous centrally hemorrhagic mass in the right anterior pararenal space, abutting both the pancreas and the duodenum ( Figure $2 \mathbf{a}$ and - Figure 2 b). At laparotomy, a $15 \times 12 \times$ $10 \mathrm{~cm}$ mass was found in the retroperito-

neum immediately posterolateral to, and intimately associated with, the second part of the duodenum ( $\bullet$ Figure 3 ). The mass was dissected with a cuff of duodenal wall, which when divided demonstrated a defect in the posterolateral wall of the duodenum, corresponding to the location of the diverticulum on endoscopy. The center of the mass had cavitated secondary to necrosis and was full of clot. This was in continuity with the lumen of the duodenum, thus giving the appearance of a bleeding diverticulum on endoscopy ( Figure 4). Surgical pa-

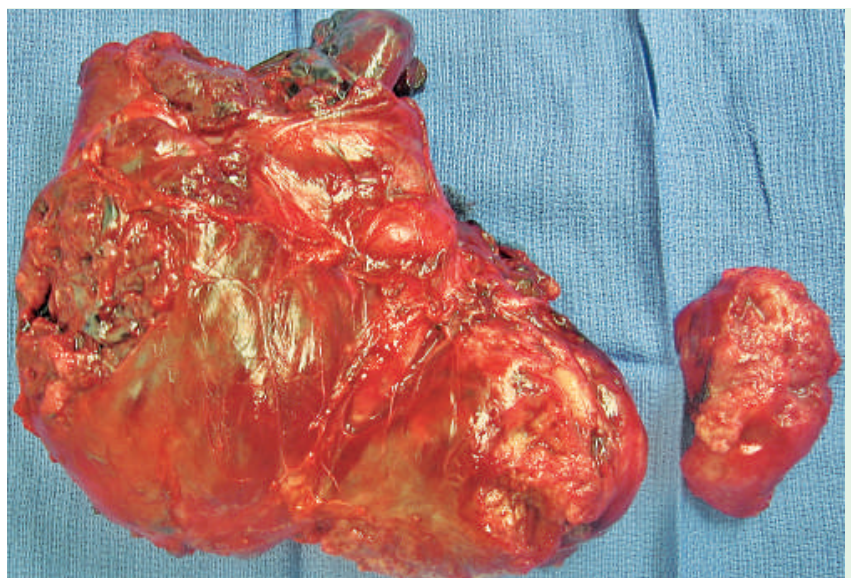

Figure 3 The gross specimen was soft, friable, and had dimensions of $15 \mathrm{~cm} \times 12 \mathrm{~cm} \times 10 \mathrm{~cm}$
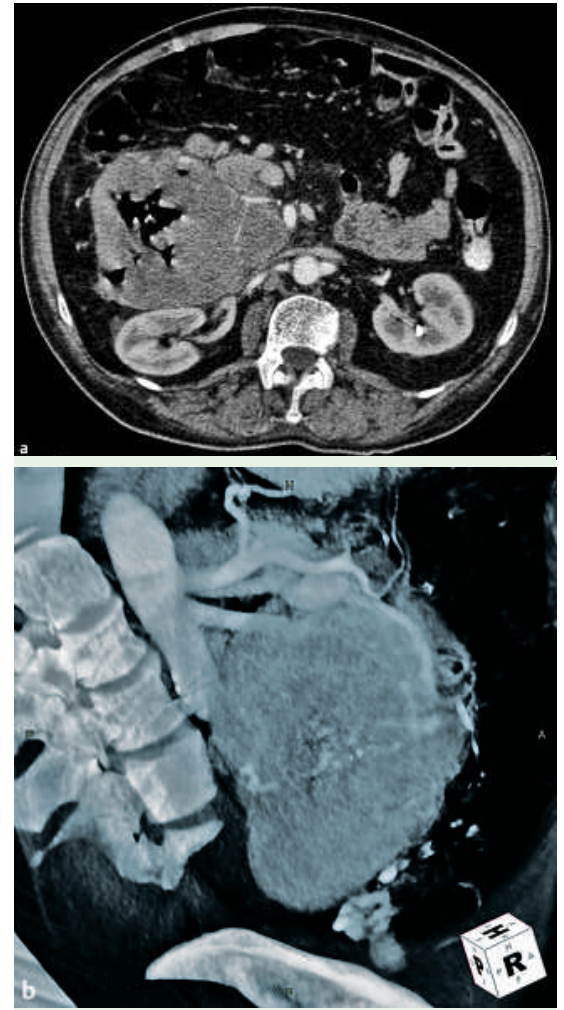

Figure 2 a CT scan of the abdomen and pelvis with contrast revealed a large $(15 \mathrm{~cm} \times 10 \mathrm{~cm})$, heterogenous, centrally hemorrhagic mass in the right anterior pararenal space, with external compression of the inferior vena cava at the renal vein. There was no evidence of a tissue interface separating the pancreas or duodenum from the mass. $\mathbf{b}$ Digital reconstruction subtraction angiography in an oblique craniocaudal view further characterized the mass as well as its blood supply directly from the aorta.

thology confirmed a spindle cell tumor consistent with high-grade gastrointestinal stromal tumor (GIST), staining positive for Vimentin and CD 117 (ckit) and negative for S100, CD56, SMA, and MSA. The patient was discharged in a stable condition, for follow-up with oncology. This constitutes the first report in the literature of a gastrointestinal stromal tumor mimicking a bleeding duodenal diverticulum.

Endoscopy_UCTN_Code_CCL_1AB_2AZ_3AB 


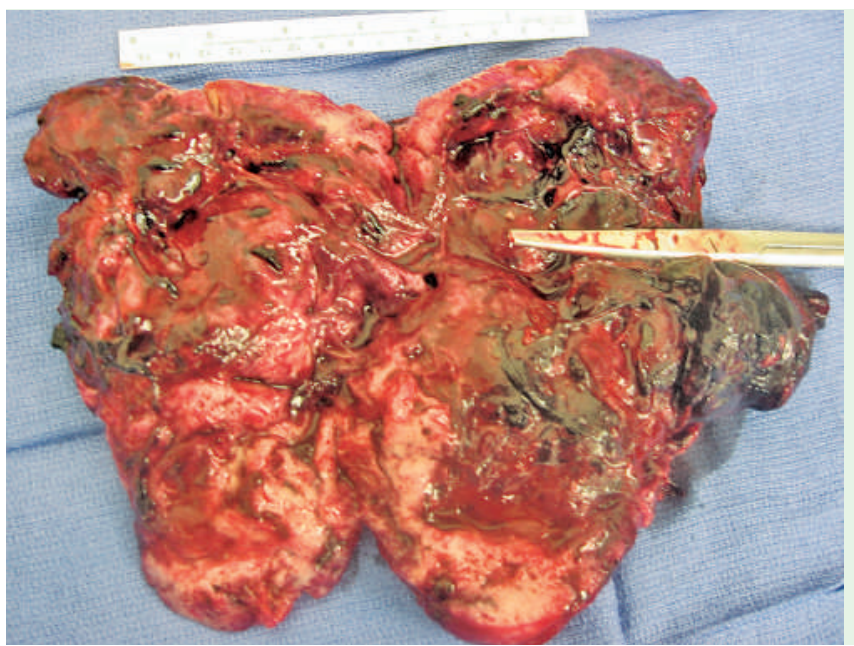

Figure 4 Transverse section of the specimen revealed central cavitation secondary to necrosis. The clot-filled necrotic center was in continuity with the lumen of the duodenum (as depicted by the instrument), thus giving the appearance of a diverticulum on endoscopy.
N. Gupta ${ }^{1}$, B. D. Schirmer ${ }^{1}$, R. Mishra ${ }^{2}$, V. M. Shami ${ }^{2}$

1 Department of Surgery, University of Virginia Health System, Charlottesville, Virginia, USA

2 Department of Medicine, University of Virginia Health System, Charlottesville, Virginia, USA

Bibliography

DOI 10.1055/s-2007-966245

Endoscopy 2007; 39: E142 -E143

(c) Georg Thieme Verlag KG Stuttgart · New York . ISSN 0013-726X

Corresponding author

\section{N. Gupta, MD}

Department of Surgery

University of Virginia Health System

PO Box 800300

Charlottesville,

VA 22908

USA

Fax: +1-434-243-5791

naren@virginia.edu 